\title{
Heart Disease New Hypotesys: Under Endogenous Toxicological Aspect
}

\author{
Luisetto $\mathbf{M}^{1 *}$, Behzad Nili $^{2}$ and Ghulam Rasool Mashori ${ }^{3}$ \\ ${ }^{1}$ Applied Pharmacologist, Independent Researcher, Italy \\ ${ }^{2}$ Innovative Pharmaceutical Product Development Specialist, USA \\ ${ }^{3}$ Department of Medical \& Health Sciences for Woman, Peoples University of Medical and Health Sciences for Women, Pakistan
}

Submission: January 18, 2018; Published: February 20, 2018

*Corresponding author: Luisetto M, Applied Pharmacologist, Independent Researcher, Italy, 29121, Email: maurolu65@gmail.com

Keywords : Cardiology research; Cardiovascular pathology; Toxicology; Gradients; Innovative therapeutic strategies

\section{Introduction}

In order to suggest new pathogenetic hypothesis in some heart disease we think is interesting to observe some biomedical literature: Can we think some endogenous toxicologic moves in some heart pathologies?

Activated platelet can trigger coronay artery spasm in some patients, and in SCD many patients not show

Anatomic abnormalities and this can be involved sudden vigorous exertion untrained subjects (also in young) in seems to show an inadequate metabolic response. Also severe arrhythmia can be due by electrolyte unbalance.

The same unbalances in autonomic nervous system can results in SCD in some subjects related to unexpected high stress condition. Can this fact be compared as an endogenous toxicological event? In example considering activated platelets like a toxic or in SCD a biochemical poisoning by inadequate rapid balances in vigorous activity in untrained? We think that observing some heart pathologies under a toxicological aspect (endogenous factors) we can verify new pharmacological strategies.

We suppose to consider this kind of event like an endogenous toxicity mechanism as new pathogenetic hypothesis. Today these kind of pathogenetic factors are not studied under a toxicological aspect, but under this approach we can think to introduce new kind of antidotes or depurative procedure.

"In past centuries physicians in some cardiologic Pathology were used to prescribe to the patient a long rest in order to recuperate health status. (There wasn't available high efficient drugs strategies and so a long rest without physical and- or psychological high stress contributes to this process). In example in oriental medicine we can see body balances strategies, and in some psychologic techniques we can see that time is relevant to re-equilibrate some conditions (acute stress).

We can think that the main factors involved are the time and long rest condition in order to have a right balance the physiologic functions. It was observed complete resolve in some pathologic situations as CHF. So we can think that a metabolic- catabolic unbalances can create this situation and the time make possible to adequately restore. In example we can see what happen in sudden death heart syndrome in untrained? Why in this condition physical training can reduce this kind of event? We can think a condition of endogen intra- toxicity time related" [1].

"We think in our opinion that in this condition is relevant to deep observe the endogen time related intra- local toxicity situation involved in some Metabolic- catabolic -electrical cell membrane - immune status and other (Kinetics). In example involved in some heart arrhythmia, epileptic status conditions, septic shock and other pathologic situation high time related (ischemic coronaric spasm et other).

In embryology, oncology, toxicology field, some heart or brain pathologies time is relevant added to local micro environment and inters -cellular communication (acute -crisis). We can consider an endogen intra- local toxicology aspect time related to deep verify some pathologic process under a new light [1].

Katritsis et al. [2] write that "CAD is the predominant causes of SCD in older athletes. Vigorous exertion can trigger cardiac arrest or SCD sudden cardiac death, especially in the untrained persons, but habitual vigorous physical exercise diminishes the risk of sudden death during the vigorous exertion. 
According the recent paper "Brain and Transmission Signal Modulation " 2017 "As observed in other scientific or research bio- medical discipline controlling some non physiological cellular activity can Results in reducing of abnormal tissue- organ activation" [3]. "There is a circadian variation in SCD sudden cardiac death. The peak incidence of SCD occurs between 6 am and noon (and is blunted by beta-blockers drugs), with a smaller peak occurring in the late afternoon for out-of-hospital VF arrests. The incidence in SCD is highest on Mondays. In the $<35$ years, the most common cause of sudden cardiac death is arrhythmia, mostly in an apparently normal heart situations.

The most common causes of sudden cardiac death are congenital abnormalities in those aged 0-13 years, primary arrhythmia in the 14-24-year age group, and CAD in those $>25$ years. In $5-20 \%$ of cases no significant cardiac abnormality is found at autopsy procedure. In a recent Danish registry report on individuals aged $<50$ years, sudden death was caused by noncardiac pathology, such as pulmonary embolism, meningitis and cerebrovascular bleeding, in $28 \%$ of cases" [4].

According to Hung et al. [5] "the Coronary artery spasm (CAS), a pathology with an intense vasoconstriction of coronary arteries (that causes total or subtotal vessel occlusion), plays an important role in myocardial ischemic syndromes including stable and unstable angina, acute myocardial infarction, and SCD sudden cardiac death.

Coronary angiography and provocative tests usually is required to establish a definitive diagnosis. But the mechanisms underlying the development of CAS coronary artery spasm are still poorly understood; CAS appears to be a multifactorial pathology but is not associated with the traditional risk factors for coronary artery disease.

The diagnosis has important therapeutic implications, as calcium antagonists, not $\beta$-blockers, are the cornerstone of medical therapy. The prognosis is generally benign; however, recurrent episodes of angina are frequently observed" [4]. And "Precipitating factors may contribute to the onset of coronary artery spasm, CAS and act in the same patient to cause angina in different conditions and situations. CAS can be precipitated by physical and/or mental stress, magnesium deficiency status, alcohol consumption, the cold pressure test, hyperventilation, the valsalva physicians maneuver, remnant lipoproteins, and the administration of pharmacological agents such as cocaine or drugs, sympathomimetic agents (epinephrine, norepinephrine), betablocking agents parasympathomimetic agents (methacholine, pilocarpine), and ergot alkaloids particularly in the morning when spontaneous CAS is most likely to occur. Activated platelets may trigger CAS by releasing vasoconstrictor substances, including thromboxane and serotonin, both of which are found to be associated with CAS [4]."

And related psychological stressing condition that can influence autonomous nervous systems (sympathetic iperactivation). "We can see that the amount of stress ant stimulus and related kinetics are relevant factors involved in some human conditions. Not only the single stress but the global amount in a limited time are involved in this situation and a chronic condition make more severe this situation"[6,7]. And related to neurologic conditions "SCD is an important but widely under-recognized consequence of stroke. Acute stroke can disturb central autonomic control, resulting in myocardial injury, electrocardiographic abnormalities, cardiac arrhythmias, and ultimately sudden death.

Experimental -clinical evidence suggests that: autonomic imbalance is more frequent after infarcts involving the insular cortex, a crucial region for the control of sympathetic and parasympathetic autonomic functions. Cardiovascular comorbidities increase the risk of cardiac morbidity and mortality after stroke.

Thus, many sudden deaths and serious non-fatal cardiac events after stroke are probably due to an interaction between cardiovascular and neurological causes. The exact mechanisms leading to sudden death remain incompletely understood" [8].

"Even if the results showed in this paper are not conclusive and that specific more experimental data are necessary we can say that under the light of the article indeed in this work but also to other works published we can think a new system "to regenerate" a valve tissue calcified. We think that adding 3 strategies we can have relevant effect.

We have seen that atherosclerotic plaques has calcific components and that EDTA is a calcium complexant agent and we have also see that interventional radiology strategy help physicians to transfer in example contrast agent in situ or medicated stents. We can think that adding these 3 strategies we can have more clinical outcomes and less complex toxicity if used active but toxic molecule as EDTA as calcium complessant in the reverse atherosclerotic plaques. Using a toxic agent but in local way we think we can obtain more useful result. A chemistry modify make possible to link the EDTA molecule to a group with easily renal clearance. Even if in past systemic therapy has not showed a real activity a new delivery systems could improve the final results. The same we can consider a new injection system to deliver the drugs into the valve tissue" [9].

\section{Discussion and Conclusion}

Can we think some endogenous toxicologic movements in some heart pathologies? Metabolic- catabolic Kinetics is relevant factor in balancing some cellular- tissue responses? Some endogenous gradients time related can be responsible in some organ failure? In toxicology field usually are high considered the external environmental factors as iatrogenic substantial but we think we must observe under toxicological methods also the endogenous intra-extra cellular local microenvironment (in paraphysiologic-pathologic situations).

In some cardio vascular pathology the time is relevant added to endogenous local micro environment and inters cellular communication status. We must consider an endogenous 
intra- local toxicology aspect time related to better verify some pathologic process under a new light. In some time related cardiovascular local metabolic-catabolic-toxic status we can observe some cellular effect resulting in global organ failure.

The time involved and kinetics aspect in resolve some temporary metabolic- catabolic gradients or the velocity involved in this process can be fundamental. The same effect related to too much rapid evolution or too slow reduction (kinetics) in balancing equilibrates some physiologic systems (in example the reduced effect showed in sports trained in SCD vs not trained, activate platelets in trigger coronary artery spams or other relevant examples). Need we to introduce more toxicological methods in some pathologies in order to better clear some relevant aspect in etiology, diagnosis and therapy?

In our opinion we must consider also endogenous factor as initiating or precipitating condition in some heart pathologies. Considering some endogenous poison like factor make possible to research related antidotes strategies. In example to think to better prevent platelet activation in a larger plates of patients or to deeply introduce diagnostic test in young people in order to better clarify the SCD pathologic cause in stressing condition (in example before starting sport activities). This approach considering a sort of a sudden endogenous poison like condition (acute or chronic conditions).

\section{Clarifications}

This paper was not written for any diagnostic or therapeutic intent, but only to produce new research hypothesis.

\section{References}

1. Luisetto M (2017) Intra- local toxicology aspect time related in some pathologic conditions. Open Acc J of Toxicol 2(3): DOI: 10.19080/ OAJT.2017.02.555586.

2. Katritsis DG, Gersh BJ, Camm AJ (2016) A clinical perspective on sudden cardiac death. Arrhythm Electrophysiol Rev 5(3): 177-182.

3. Luisetto M (2017) Short communication brain and transmission signal modulation. Theranostics of Brain Disorder 1(1): 01.

4. Luisetto M, Luca C, Farhan AK, Ghulam RM (2017) Sudden heart pathology-a new research hipotesys. J Cardiol \& Cardiovasc Ther 8(1): $1-3$.

5. Hung MJ, Hu P, Hung MY (2014) Coronary artery spasm: review and update. Int J Med Sci 11(11): 1161-1171.

6. Luisetto M, Nili BA, Mashori GR (2018) Mindset kinetics-under toxicological aspect. Psychology and Behavioral Science 8(2): 1-2.

7. Myerburg RJ, Junttila MJ (2012) Sudden cardiac death caused by coronary heart disease. Circulation 125(8): 1043-1052.

8. Sörös P, Hachinski V (2012) Cardiovascular and neurological causes of sudden death after ischaemic stroke. Lancet Neurol 11(2): 179-188.

9. Luisetto M, Nili-Ahmadabadi BM, Mashori GR (2017) Surgery and new pharmacological strategy in some atherosclerotic chronic and acute conditions. Arch Surg Clin Res 1: 42-48.

\begin{tabular}{|l|}
\hline \multicolumn{1}{|c|}{$\begin{array}{c}\text { Your next submission with Juniper Publishers } \\
\text { will reach you the below assets }\end{array}$} \\
- Quality Editorial service \\
- Swift Peer Review \\
- Reprints availability \\
- E-prints Service \\
- Manuscript Podcast for convenient understanding \\
- Global attainment for your research \\
- Manuscript accessibility in different formats \\
( Pdf, E-pub, Full Text, Audio) \\
- Unceasing customer service \\
Track the below URL for one-step submission \\
https://juniperpublishers.com/online-submission.php \\
\hline
\end{tabular}

